\title{
Influence of modern technologies used in rehabilitation on selected functional parameters of the spine of patients with low back pain
}

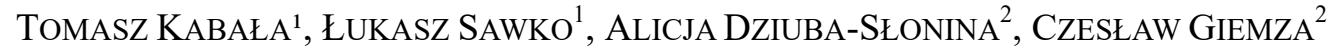 \\ ${ }^{1}$ Prophylaxis and Rehabilitation Centre "Creator", Wrocław, Poland. \\ ${ }^{2}$ Faculty of Physiotherapy, University School of Physical Education in Wrocław, Poland.
}

\begin{abstract}
Purpose: The aim of the study was to examine influence of modern technologies used in the DBC method (Documentation Based Care) on the selected functional parameters of the spine of patients with low back pain. Methods: The research group comprised of 196 patients randomly divided into two groups. Patients in Group I were subjected to DBC treatment with modern rehabilitation equipment, while patients in Group II underwent the traditional form of therapeutic exercise. All subjects included in the study suffered from chronic low-back pain lasting for a period of at least three months. All the symptoms were non-specific. Results: The results presented in the article unequivocally reveal that patients who underwent DBC treatment achieved better, statistically significant improvement in spinal functionality. Conclusions: The modern technologies introduced to the treatment had a considerable impact on the selected functional parameters of the spine of patients with low back pain. The DBC method used as a rehabilitation program for low back pain had a statistically significant influence on the functional parameters of the spine.
\end{abstract}

Key words: modern technologies, rehabilitation, back pain, DBC method, active therapy

\section{Introduction}

Low back pain syndrome is one of the most frequently observed lifestyle diseases and the main cause of disability in the world [10]. Approximately $80 \%$ of adults have at least one episode of back pain in their lifetime. Most of these episodes are transient in nature. However, about $10 \%$ of all people develop chronic pain and disability beyond three months. The high incidence of pain and its recurrence are mirrored in the number of lost working days and inability to work [9], [14].

A wide range of treatment modalities have been introduced over the years with differing degrees of effectiveness in low back pain treatment. Many studies have shown that active rehabilitation based on therapeutic exercises is effective in treatment of spinal disorders [7], [16], [17]. The development of modern technologies allows for the introduction of many state-of-the-art diagnostic tests and treatments, as well as the search for innovative and technologically-developed solutions supporting the treatment of back pain. An example of such therapy is the DBC method (Documentation Based Care), which is the result of the cooperation of doctors, physiotherapists, engineers and biomechanics [1], [11], [23]. Their combined effort led to creating modern DBC devices based on innovative technological solutions, which are used in diagnostics and the treatment of patients with spinal disorders. Goniometers installed in the devices allow for measuring of the range of spinal motion, while dynamometers test muscle strength. Modern technological solutions involve making individual adjustments of particular devices to specific aspects of patients, and saving these settings on a personal magnetic card. This

\footnotetext{
* Corresponding author Tomasz Kabała, Prophylaxis and Rehabilitation Centre "Creator”, ul. Lotnicza 37, 54-154 Wrocław, Poland. Phone: +48 500113 516, e-mail: tomasz.kabala@creator.wroc.pl

Received: April 15th, 2020

Accepted for publication: September 22nd, 2020
} 
enables the patients to perform exercise and be measured under constant conditions. This solution provides precise body stabilization and limits the activity of muscles supporting spinal movements whose range is precisely defined by a regulating mechanism.

The aim of the study was to examine the influence of modern technologies used in the DBC method on the efficacy of rehabilitation of patients with low back pain. Researchers hypothesized that the use of such tools and treatment can improve the selected functional parameters of the lumbar spine in patients with this pain syndrome.

\section{Materials and methods}

The research group comprised of one-hundred ninety-six patients randomly divided into two groups. Patients in Group I were subjected to DBC treatment with modern rehabilitation equipment, while patients in Group II underwent the traditional form of therapeutic exercise. The mean age of patients in Group I was 56.3 and Group II - 58.1. There were 50 women and 46 men in Group I and 54 women and 46 men in Group II (Table 1). Two-hundred plastic capsules were put in a container, one-hundred of each were designated with Group I and Group II. The patients qualified for the study, after they have been registered, drew a capsule from the container which assigned them to a particular group. Each research group was comprised of onehundred participants. Ninety-six patients completed the treatment in Group I, four participants did not complete the therapy due to reasons beyond our control. All patients from Group II completed the rehabilitation.

Table 1. Characteristics of the group

\begin{tabular}{|l|c|c|}
\hline & Group I & Group II \\
\hline Number of patients & 96 & 100 \\
\hline Women & 50 & 54 \\
\hline Men & 46 & 46 \\
\hline Mean age [years] & 56.3 & 58.1 \\
\hline Height [cm] & 166.1 & 163.7 \\
\hline Body weight [kg] & 88.1 & 84.0 \\
\hline
\end{tabular}

The rehabilitation program for Group I lasted 90 minutes, twice a week for 6 weeks. Group II was subjected to traditional treatment comprised of exercises. The program lasted 60 minutes, twice a week for 6 weeks.

All subjects included in the study suffered from chronic low-back pain lasting for a period of at least three months, with a VAS score greater than $30 \mathrm{~mm}$.
All the symptoms were non-specific. As described by the WHO, non-specific pain symptoms are those of an unknown etiology, and of no distinct tissue damage responsible for causing the pain. None of the patients reported radicular pain (i.e., negative results of the Lasegue test, no sensory disorders and paresis).

The exclusion criteria were as follows:

- severe condition (infections, nerve root entrapment with intolerable pain),

- pain radiating down the leg below the gluteal crease,

- recent spinal fracture,

- spinal instability excluding active rehabilitation,

- severe osteoporosis,

- oncology diseases,

- mental disorder/severe psychological disturbance.

Treatment of the pain aims to improve the function of the spine by increasing its mobility, strengthening paraspinal muscles, reducing pain (breaking the "vicious circle of pain" associated with not using the spine), thus reducing difficulties caused by pain. Its objective is also to educate patients on taking care not to overload the spine during daily activities.

Patients in Group I were subjected to DBC active rehabilitation with specially designed equipment, running diagnostics and performing exercises of selected muscle groups of the lumbo-thoracic spine region (multifidus and erector spinae muscles, transverse abdominal muscle, rectus abdominis muscle, oblique muscles of abdomen, quadratus lumborum muscle). DBC devices were designed to carry out spinal treatment under conditions of repetitive, dynamic loading while performing controlled flexion, extension, rotation and lateral flexion of the lumbo-thoracic spine. Each device can be adjusted to the individual parameters of a patient (seat height and foot-platform height). Such personal settings are stored on a private card, enabling measurements to be taken and exercises to be performed under constant conditions. The devices are equipped with a locking mechanism, which eliminates compensatory movement in other parts of the spine and muscle. The treatment applied in the research involved performing exercises with an individually adjusted range of motion, loading and number of repetitions. The first period of treatments was based on exercises with low loading, the objective being to improve mobility. Special focus was put on teaching proper co-ordination and control of spinal movement. Next, pressure on spinal muscle endurance was gradually increased. Loading was successively increased so that at the fourth week a subjectively strenuous load was applied for the first time but within the pain tolerance of the individual patient. 
The devices applied in the DBC method have a dual function: they are used in both rehabilitation programs and clinical diagnostics. The goniometers built into the devices (Fig. 1) provide accurate measurements of range of motion. Each device also measures the moment of force in relative muscles. Additionally, the bioelectric activity of selected muscles (sEMG) can be measured.

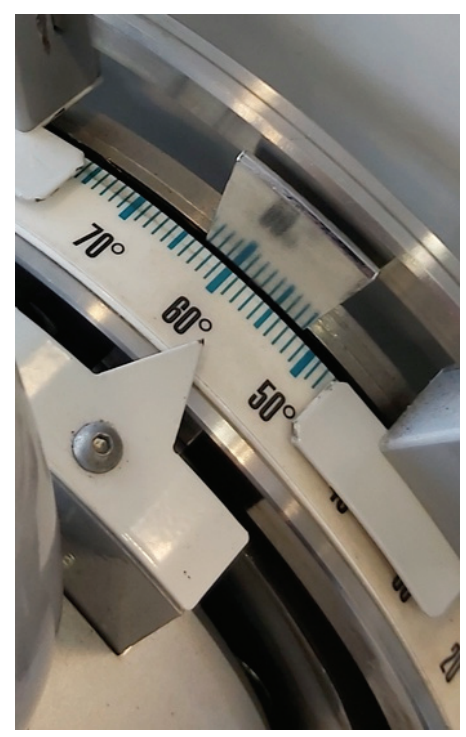

Fig. 1. Goniometer built into DBC device

The following treatment devices are used in DBC active low back pain therapy:

- LTE - device for measuring: range of motion in the lumbar-thoracic spine in the sagittal plane (flexion extension in a sitting position), extension moments of the same area of the spine and active resistance exercises strengthening spine extensors (Fig. 2);

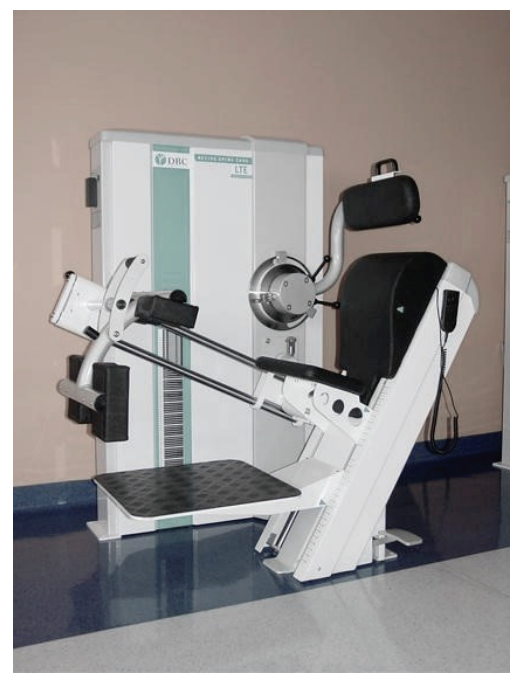

Fig. 2. LTE - DBC device for diagnostics and active resistance exercise of lumbar thoracic spine extension
- LTF - device for exercising with resistance and measuring flexion moments of the lumbar-thoracic spine in the sagittal plane in the sitting position;

- LTR - device for measuring: the range of rotational movement and moments of force in the lumbar-thoracic spine in the transverse plane and performing resistance exercises of transverse abdominal muscles through rotational movement of the lower body in the sitting position (Fig. 3);

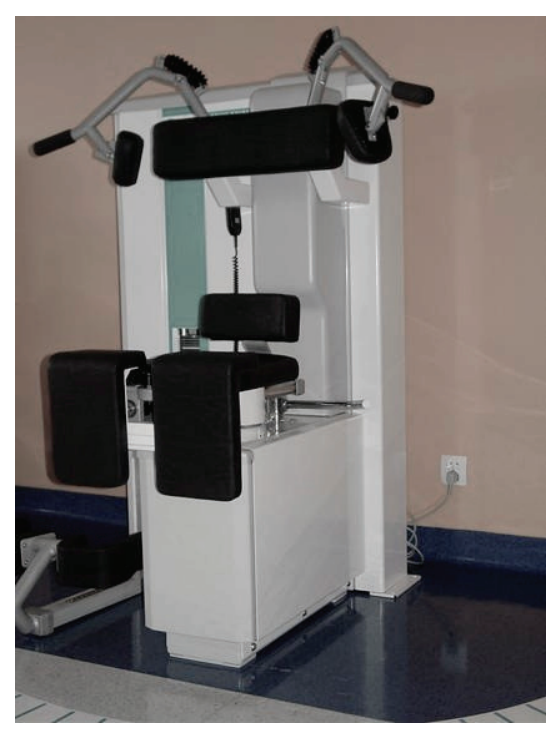

Fig. 3. LTR - DBC device for diagnostics and active resistance exercise of lumbar thoracic spine rotation

- LTL - device for measuring: range of motion and moments of force in lateral or side-bending of the lumbar-thoracic spine and strengthening lateral spine flexors in the sitting position (Fig. 4) [5];

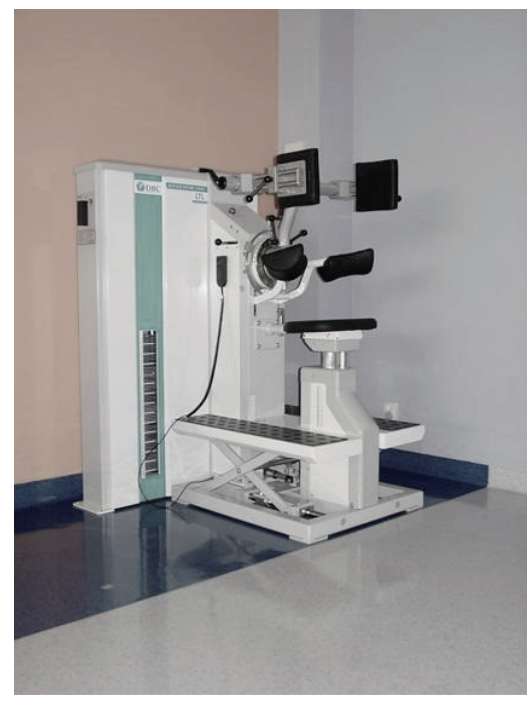

Fig. 4. LTL - DBC device for diagnostics and active resistance exercise of lumbar thoracic spine lateral flexion 
Patients in Group II performed exercises used in traditional rehabilitation programs in the case of chronic back pain: exercises strengthening abdominal and dorsal muscles in low positions (supine position, prone position, lateral position and supported kneeling), exercises increasing the mobility of the spine, pelvis and lower limbs, exercises for correct body posture and relaxation exercises. All exercises were applied within the pain tolerance of the individual patient.

At the beginning of each training session, both Groups carried out a 15-min warm-up using a cycle ergometer and equipment, such as a stepper. Warm-up was included in the time of the training session. The warm-up (pace, resistance) were adjusted to the capacity of the individual patient. Additionally, patients in Group I and Group II performed stretching, relaxation and functional exercises (imitating activities typical for a person exercising in everyday life). The two programs were also enriched through education on ergonomics. The researchers used DBC devices to evaluate the efficacy of rehabilitation in both groups before (Test 1) and after the treatment (Test 2). Measurement of the bioelectrical activity (sEMG) of erector spinae muscle in the lumbar area for the right and left sides were performed (Fig. 5). Measurements were taken in two positions: unconstrained, standing position and maximum flexion.

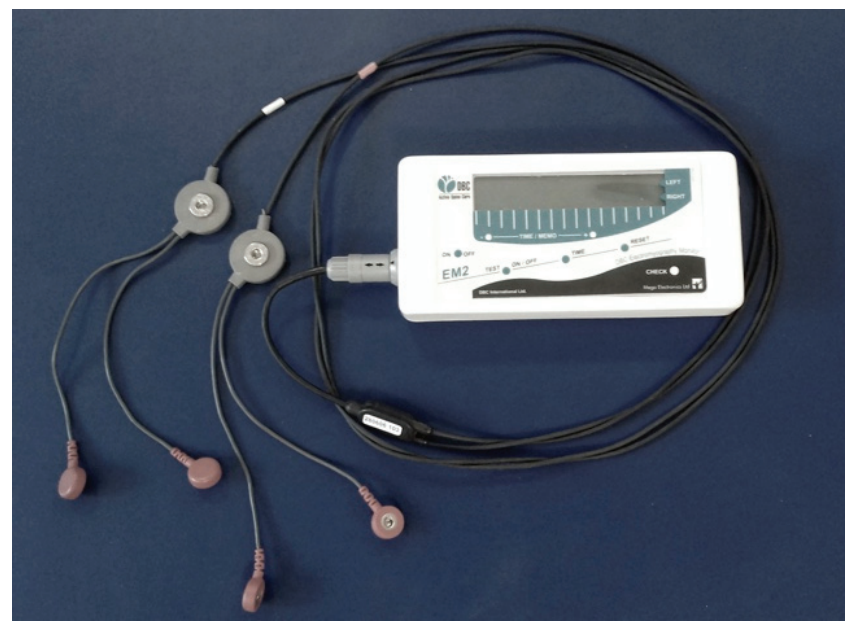

Fig. 5. DBC EMG Monitor

Range of motion was measured for the lumbarthoracic spine in: flexion, extension, lateral flexion to the right, lateral flexion to the left, right rotation, left rotation. The moment of force was measured for: lumbar extensors, lumbar flexors, lumbar spine rotators to the left, lumbar spine rotators to the right, lumbar flexors to the left, lumbar flexors to the right (Fig. 6).

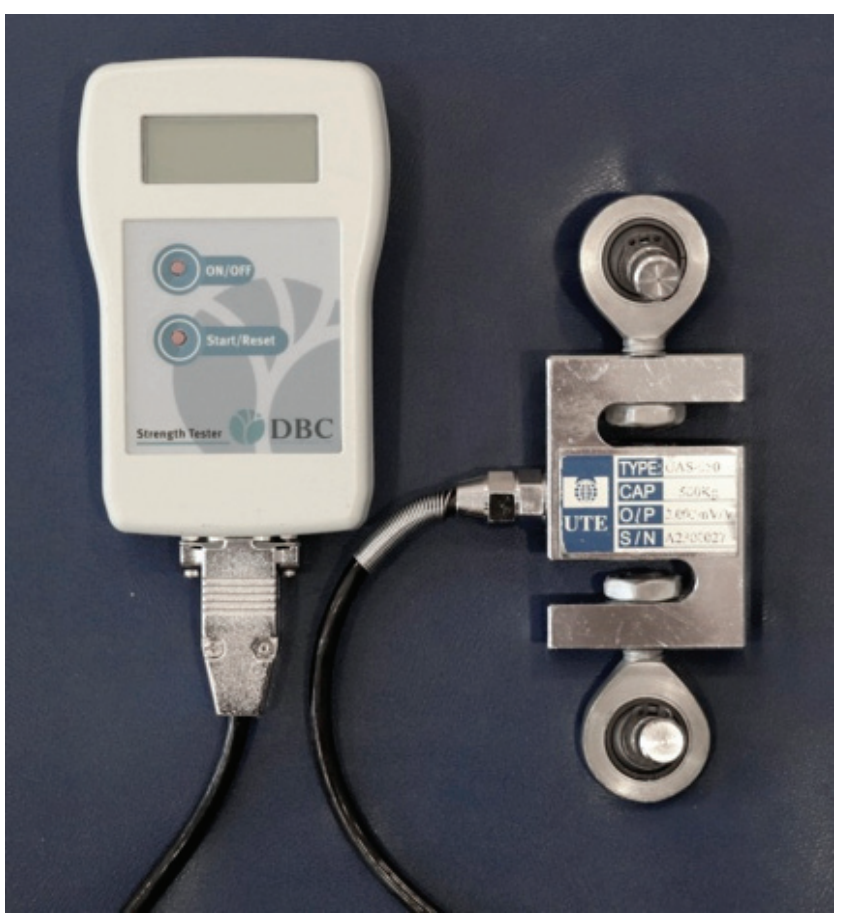

Fig. 6. DBC Strength Test

The schematics of the research protocol was presented in a flow chart (Fig. 7).

The sequence of the stages of the research

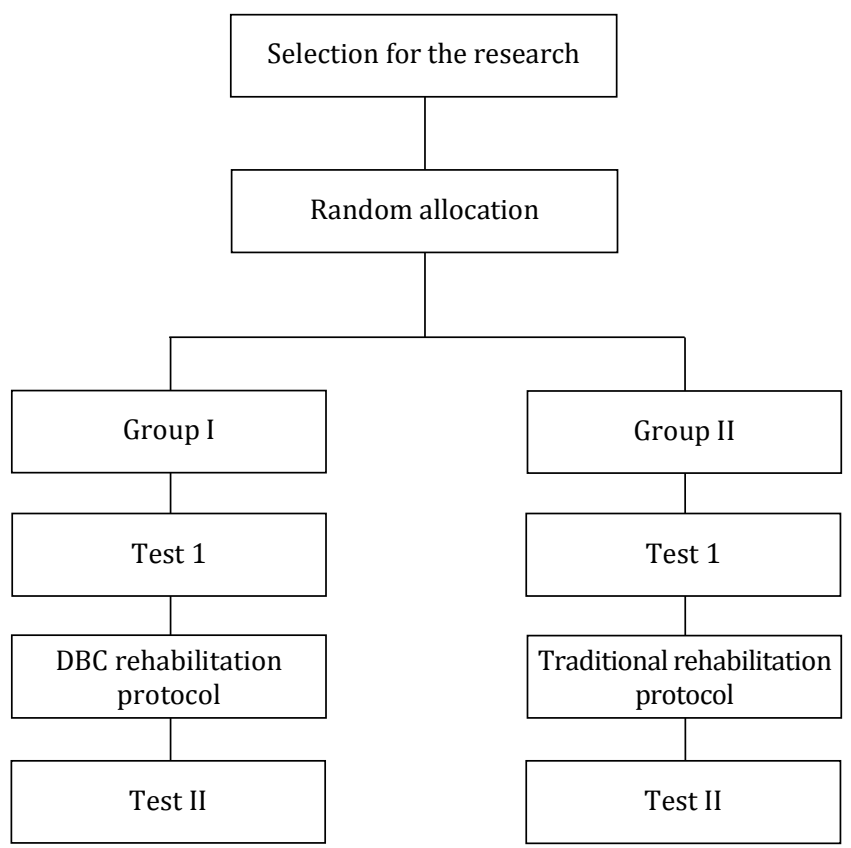

Fig. 7. Description of research protocol 


\section{Results}

Statistical analysis was carried out using Statistica 12 PL software. The distribution of the data set was screened for normality using the Shapiro-Wilk test. The significance of intergroup differences was examined with the Mann-Whitney $U$-test. The effectiveness of the applied therapy within each group was measured with the Wilcoxon signed-rank test. The results were considered significant for $p<0.05$. Preliminary statistical analysis did not show significant differences in the examined parameters between male and female patients, therefore, results are presented for the whole group. There were also no statistically significant differences between the studied groups. This allows for the conclusion that patients in both groups commenced the therapy at the same level of functionality of the lumbar spine.

The results presented in Tables 2-4 unequivocally reveal that patients who underwent DBC treatment achieved better, statistically significant improvement in spinal functionality. There was a significant increase in the range of spinal motion and muscle strength in the lumbar spine. The functional potential of muscles in the lumbar region of the spine was also significantly reduced, indicating the normalization of muscle tensions in this area. Results of the patients in Group II, who were subjected to traditional rehabilitation program, also improved, however, they were not statistically significant.

The greatest improvement in Group I was observed in the values of the functional potential of the lumbar extensor to the left in a standing position

Table 2. Mean values and standard deviation of the functional potential of spine extensors $[\mu \mathrm{V}]$

\begin{tabular}{|l|c|c|c|c|c|c|c|c|}
\hline \multirow{2}{*}{ sEMG } & \multicolumn{5}{|c|}{ Group I } & \multicolumn{4}{c|}{ Group II } \\
\cline { 2 - 9 } & Test 1 & Test 2 & $p$ & $\%$ & Test 1 & Test 2 & $p$ & $\%$ \\
\hline $\begin{array}{l}\text { Standing position } \\
(\text { Left side) }\end{array}$ & $18.2( \pm 3.9)$ & $10.1( \pm 4.1)$ & 0.001 & 44.5 & $19.6( \pm 6.8)$ & $18.3( \pm 5.9)$ & 0.149 & 6.6 \\
\hline $\begin{array}{l}\text { Standing position } \\
(\text { Right side) }\end{array}$ & $19.2( \pm 4.1)$ & $12.9( \pm 6.3)$ & 0.003 & 32.8 & $20.1( \pm 9.1)$ & $17.3( \pm 8.9)$ & 0.201 & 13.9 \\
\hline $\begin{array}{l}\text { Maximum flexion } \\
\text { (Left side) }\end{array}$ & $31.0( \pm 11.1)$ & $18.0( \pm 7.1)$ & 0.000 & 41.9 & $33.1( \pm 11.8)$ & $29.3( \pm 14.1)$ & 0.654 & 11.5 \\
\hline $\begin{array}{l}\text { Maximum flexion } \\
\text { (Right side) }\end{array}$ & $29.3( \pm 11.8)$ & $19.3( \pm 5.9)$ & 0.000 & 34.1 & $30.8( \pm 11.9)$ & $27.3( \pm 14.1)$ & 0.512 & 11.4 \\
\hline
\end{tabular}

Table 3. Mean values and standard deviation of the range of motion for the lumbar spine $\left[{ }^{\circ}\right]$

\begin{tabular}{|l|c|c|c|c|c|c|c|c|}
\hline \multirow{2}{*}{ Range of motion } & \multicolumn{4}{|c|}{ Group I } & \multicolumn{4}{c|}{ Group II } \\
\cline { 2 - 9 } & Test 1 & Test 2 & $p$ & $\%$ & Test 1 & Test 2 & $p$ & $\%$ \\
\hline Extension & $26.8( \pm 9.7)$ & $33.5( \pm 8.2)$ & 0.000 & 20.0 & $24.3( \pm 9.2)$ & $26.2( \pm 8.1)$ & 0.065 & 7.3 \\
\hline Flexion & $39.9( \pm 10.9)$ & $50.4( \pm 9.4)$ & 0.000 & 20.8 & $41.0( \pm 12.1)$ & $41.9( \pm 9.9)$ & 0.081 & 2.1 \\
\hline Right rotation & $29.3( \pm 7.1)$ & $31.9( \pm 5.5)$ & 0.003 & 8.2 & $28.9( \pm 9.3)$ & $30.1( \pm 8.4)$ & 0.077 & 3.9 \\
\hline Left rotation & $28.6( \pm 9.2)$ & $38.0( \pm 8.4)$ & 0.002 & 24.7 & $33.1( \pm 16.9)$ & $33.7( \pm 12.1)$ & 0.165 & 1.8 \\
\hline Flexion to the right & $45.0( \pm 6.9)$ & $50.2( \pm 5.3)$ & 0.000 & 10.4 & $45.9( \pm 12.9)$ & $46.2( \pm 8.6)$ & 0.202 & 0.65 \\
\hline Flexion to the left & $46.6( \pm 7.0)$ & $51.7( \pm 5.5)$ & 0.000 & 9.9 & $46.9( \pm 11.1)$ & $48.3( \pm 15.0)$ & 0.081 & 2.9 \\
\hline
\end{tabular}

Table 4. Mean values and standard deviation of muscle strength $[\mathrm{Nm} / \mathrm{kg}]$

\begin{tabular}{|l|c|c|c|c|c|c|c|c|}
\hline \multirow{2}{*}{ Muscle strength } & \multicolumn{4}{|c|}{ Group I } & \multicolumn{4}{c|}{ Group II } \\
\cline { 2 - 9 } & Test 1 & Test 2 & $p$ & $\%$ & Test 1 & Test 2 & $p$ & $\%$ \\
\hline Extension & $826.5( \pm 404.5)$ & $1116.7( \pm 481.2)$ & 0.001 & 26 & $911.1( \pm 367.0)$ & $961.0( \pm 249.2)$ & 0.731 & 5.2 \\
\hline Flexion & $456.8( \pm 339.0)$ & $654.5( \pm 370.1)$ & 0.000 & 30.2 & $483.2( \pm 103.5)$ & $501.1( \pm 206.1)$ & 0.697 & 3.6 \\
\hline Right rotation & $197.3( \pm 124.8)$ & $353.4( \pm 213.2)$ & 0.003 & 44.2 & $201.3( \pm 98.3)$ & $260.1( \pm 100.3)$ & 0.324 & 22.6 \\
\hline Left rotation & $221.8( \pm 151.5)$ & $345.4( \pm 234.2)$ & 0.001 & 35.8 & $209.1( \pm 101.4)$ & $260.3( \pm 99.8)$ & 0.332 & 19.7 \\
\hline Flexion to the right & $625.7( \pm 359.0)$ & $913.5( \pm 410.7)$ & 0.000 & 31.5 & $598.7( \pm 328.1)$ & $631.1( \pm 229.3)$ & 0.821 & 5.1 \\
\hline Flexion to the left & $543.0( \pm 269.2)$ & $920.7( \pm 492.3)$ & 0.000 & 41 & $612.2( \pm 328.1)$ & $681.9( \pm 391.9)$ & 0.796 & 10.2 \\
\hline
\end{tabular}


(Table 2) while the smallest improvement in the rotation to the right (Table 3). Similarly, in Group II, the greatest improvement was achieved in the strength of the lumbar spine rotators to the right (Table 4) and the least improvement was observed in the lateral flexion to the right (Table 3).

\section{Discussion}

The results of the DBC approach clearly show the positive influence of modern technologies on the functional parameters of low back pain. All objective functional parameters of the lumbar spine analysed in this study significantly improved. The results obtained by the group which underwent traditional treatment also improved from a clinical perspective, however, not at statistically significant levels.

Patients engaged in this rehabilitation program based on the same DBC concept used the same devices but its components (load, range of motion, pace of exercise) were individually selected. Lumbar treatment was planned based on patients' diagnosis, range of motion, muscle strength and interview. The treatment applied involved performing exercises with an individually adjusted range of motion and loading. Treatments in the first week began with low loading and aimed at improving mobility. The main emphasis was on teaching patients proper coordination and control of spinal movement. When segmental motion and improved motor control were achieved, the pressure on spinal-muscle endurance was gradually increased. Loading was successively increased so that at the fourth to fifth week (six-week program) a subjectively strenuous load was applied for the first time but within the pain tolerance of the individual patient [2].

Back pain, especially in the lumbar region of the spine, has a negative impact on general health and affects the ability to work [13], [15]. Such cases can be observed in health care professionals. The pain is caused by forced body position during the work, which leads to muscle fatigue of the spinal extensors and their disbalance (asymmetry). Also asymptomatic occurrence of asymmetry of tension and disbalance of the spinal extensors can lead to the overload and back pain in the future [24]. Chronic pain is often related to a significant limitation in physical and mental fitness. Pain connected with this ailment substantially limits the mobility of patients and develops the behaviour of avoiding movement in order not to provoke or exacerbate the pain. Long-lasting and severe pain can lead to depression [4], [21]. Physical activity limited by pain may cause many health problems related not only to motor system disorders but also other important physiological systems. The impairment of cardiovascular, respiratory or motor systems influence the impairment of many activities necessary for independent daily functioning. They also reduce the physical capacity of these people [19]. Thus, ongoing research is looking to find efficient methods and ways to reduce existing ailments and limit their reoccurrence. One of these methods is DBC. The modern and unique technology applied in this treatment has a significant, positive impact on the results of therapy, as proven by the results obtained in this study. The aim of this method of rehabilitation is to develop motor control of spinal movement, increase spinal-muscle endurance and recreate segmental motion. The individually selected positions on the equipment, range limiters and correct loading ensure that the exercises performed are painless. The patient has the chance to overcome their fear of pain during any painful movement they may have experienced previously. It should be remembered that a patient who has experienced chronic pain has the feeling stored in their memory. Numerous studies existing which investigate and describe this phenomenon [3]. This also involves defensive mechanisms aimed at the avoidance of movement based on the fear of pain. The DBC approach aims to break the vicious cycle of pain and alter avoidance behaviours. DBC treatments are not only exercises, but also activities based on educating patients about performing daily activities without spinal overload.

The literature review shows that active rehabilitation based on therapeutic exercises is considered to be one of the most effective in spinal disorders. However, which of the therapeutic exercises is most effective has yet to be determined. Standard exercises may also improve the tested parameters, however, according to the authors, they lack the tools to precisely monitor the progress of therapy, the selection of loads, range of motion and focus on movement in specific parts of muscles [12], [18], [20]. Without such a possibility, the results obtained in Group I were better. There is a considerable body of literature confirming the efficiency of rehabilitation in the treatment of spinal pain syndrome [12], [18], [20]. The present study confirmed these findings. The results of both groups showed an improvement in the functional parameters of the lower back. Nevertheless, the outcome of the therapeutic DBC concept confirms that search, development and application of new, innovative technologies in rehabilitation have a remarkable influence on the improvement of the efficacy of an applied treatment. Another instance supporting the effective- 
ness of the technological solutions facilitating healing used in this research may be the use of cryotherapy chambers. Such solutions are based on the use of extremely low temperatures. The effectiveness of cryotherapy in the treatment of pain in the motor system, including lower back, has been confirmed by many researchers [6], [22].

\section{Conclusions}

The evaluation of efficacy of the method presented in this study was based on objective parameters: range of motion, electromyography and moment of force. Diagnostics in the DBC method also involves a number of subjective parameters. To name a few, they are: pain, functional limitations, level of depression, painrelated difficulties or faith in recovery. The further research is ongoing on the parameters described and articles are being prepared to be published.

The modern technologies introduced to the treatment had a considerable impact on the efficacy of the rehabilitation of patients with low back pain. The DBC method used as a rehabilitation program for low back pain had a statistically significant influence on the selected functional parameters of the spine.

\section{References}

[1] ANUAR K., Sim E., A retrospective study on a specialized intensive exercise program for patients with low back pain, Physiotherapy Singapore, 2003, 6 (1), 7-13.

[2] Barocha K., KiKowski Ł., Barocha M., Kabata T., Neumann-Podczaska A., Assessment of Therapeutic Effects on Documentation Based Care Treatment in Pain and Mobility of the Lumbosacral Spine in Degenerative Disease, Acta Balneol., 2017, 150 (4), 328-335.

[3] Campello M., Nordin M., Weiser S., Physical exercise and low back pain, Scan. J. Med. Sci. Sports, 1996, 6, 63-72.

[4] EllegaArd H., Pedersen B.D., Stress is dominant in patients with depression and chronic low back pain, A qualitative study of psychotherapeutic interventions for patients with non-specific low back pain of 3-12 months, BMC Musculoskelet. Disord., 2012, 13, 166-169.

[5] Giemza C., KabaŁa T., DBC - a system of active therapy of the spine, Fizjoterapia, 2007, 15(1), 55-66.

[6] Giemza C., MatczaK-Giemza M., Ostrowska B., Bieć E., DOLIŃSKI M., Effect of cryotherapy on the lumbar spine in elderly men with back pain, Aging Male, 2014, 17 (3), 183-188.

[7] Gordon R., Bloxham S., A Systematic Review of the Effects of Exercise and Physical Activity on Non-Specific Chronic Low Back Pain, Healthcare (Basel), 2016, 4 (2), 22.

[8] GREENLEAF J.E., Some evils of prolonged bed-rest deconditioning, Medicina Sportiva, 2001, 5, E77-E95.
[9] Hoy D., Bain C., Williams G., A systematic review of the global prevalence of low back pain, Arthritis Rheum., 2012, 64, 2028-2037.

[10] Hoy D., MArch L., Brooks P., The global burden of low back pain: estimates from the Global Burden of Disease 2010 study, Ann. Rheum. Dis., 2014, 73, 968-974.

[11] KabaŁa T., GiEmZa C., Efficiency of active therapy for low back pain in elderly men, J. Back Musculoskelet. Rehabil., 2019, Pre-press: 1-7, DOI: 10.3233/BMR-171072.

[12] Malfliet A., Ickmans K., Huysmans E., Coppieters I., Willaert W., Bogaert W.V., Best Evidence Rehabilitation for Chronic Pain. Part 3: Low Back Pain, J. Clin. Med., 2019, 8 (7), 1063.

[13] Malmivaara A., Hakkinen U., Aro T., Heinrichs M.L., Koskenniemi L., KuOSMA E. at al., The treatment of acute low back pain-bed rest, exercises, or ordinary activity?, N. Eng. J. Med., 1995, 332 (6), 351-355.

[14] ManchiKanti L., Singh V., Datta S., Cohen S.P., Hirsch J.A., Comprehensive review of epidemiology, scope, and impact of spinal pain, Pain Physician, 2009, 12, E35-E70.

[15] Manchikanti L., Singh V., Falco F.J.E., Benyamin R.M., HIRSCH J.A., Epidemiology of Low Back Pain in Adults, Neuromodulation, 2014, 17, 3-10.

[16] Mannion A.F., Taimela S., Muntener M., DvoraK J., Active treatment for chronic low back pain. Part 1. Effects on back muscle activation, fatigability, and strength, Spine, 2001, 26 (8), 897-908.

[17] Mannion A.F., Muntener M., Taimela S., DvoraK J., Comparison of three active therapies for chronic low back pain: results of a randomized clinical trial with one-year follow up, Rheumatology, 2001, 40 (7), 772-778.

[18] Owen PJ., Miller C.T., Mundell N.L., VerswiJveren S.J.J.M., TAGLIAFERRI S.D., BRISBY H. et al., Which specific modes of exercise training are most effective for treating low back pain?, Network Metaanalysis, Br. J. Sports Med., 2019, 0, $1-12$.

[19] Pryce R., Johnson M., Goytan M., Passmore S., BerRington N., KriellaARS D., Relationship between ambulatory performance and self-rated disability in patients with lumbar spinal stenosis, Spine, 2012, 37, 1316 $-1323$.

[20] Rice D., McNair P., Huysmans E., Letzen J., Finan P., Best Evidence Rehabilitation for Chronic Pain. Part 5: Osteoarthritis, https://www.ncbi.nlm.nih.gov/pubmed/31652929J Clin. Med., 2019, 8(11), 1769.

[21] SiPKO T., KUCZYŃSKI M., Intensity of chronic pain modifies postural control in low back patients, Eur. J. Pain, 2013, 17 (4), 612-620.

[22] Stanek A., Cieślar G., Matyszkiewicz B., Rozmus-KucZia I., Sieroń-Stoltny K., Subjective estimation of therapeutic efficacy of whole-body cryotherapy in patients with ankylosing spondylitis, Balneologia Polska, 2005, 1-2, 24-32.

[23] Taimela S., Diederich C., Hubsch M., Heinricy M., The role of physical exercise and inactivity in pain recurrence and absenteeism from work after active outpatient rehabilitation for recurrent or chronic low back pain: a follow up study, Spine, 2000, 25 (14), 1809-1816.

[24] Zyznawska J., Frankowski G., Wodka-Natkaniec E., KolomańsKa D., BoczoŃ K., Kulesa-MrowiecKa M., Disbalance and fatigue of the spinal extensors as one of the causes of the overload disease of the lumbar spine, Acta Bioeng. Biomech., 2019, 21 (3), 119-125. 